\title{
POTENTIAL APPLICATION OF MAGNESIUM DIBORIDE FOR ACCELERATOR MAGNET APPLICATIONS*
}

\author{
L. D. Cooley, C. B. Eom, E. E. Hellstrom, and D. C. Larbalestier \\ Applied Superconductivity Center, University of Wisconsin, Madison, Wisconsin 53706 USA
}

\begin{abstract}
This paper reviews the potential of the newly discovered and unexpected $39 \mathrm{~K}$ superconductor $\mathrm{MgB}_{2}$ as a material for accelerator magnet strands. Unlike the high temperature cuprate superconductors, intergranular current flow in $\mathrm{MgB}_{2}$ is not obstructed by weak links, removing one serious obstacle to the fabrication of wires. The critical current density $J_{c}$ exceeds $10^{5} \mathrm{~A} / \mathrm{cm}^{2}$ in $1 \mathrm{~T}$ field at $4.2 \mathrm{~K}$ and in self-field at $20 \mathrm{~K}$. However, the compound is anisotropic and the perpendicular upper critical field $H_{c 2 \perp}$ in present bulk samples is comparable to or less than that in Nb47wt.\%Ti at $4.2 \mathrm{~K}$. Thin films have shown significantly higher $J_{c}$ and $H_{c 2}$. Magnetization $J_{c}$ values at $4.2 \mathrm{~K}$ exceed $10^{6} \mathrm{~A} / \mathrm{cm}^{2}$ in many reports, and approach $10^{7} \mathrm{~A} / \mathrm{cm}^{2}$ for films with $T_{c}$ close to $39 \mathrm{~K}$. In alloyed films with small grain size and high resistivity, the irreversibility field $H^{*}\left(\sim 0.85 H_{c 2}\right)$ is $16 \mathrm{~T}$ at $4.2 \mathrm{~K}$ in perpendicular field (and double that in parallel field), and $J_{c}$ exceeds $10^{5} \mathrm{~A} / \mathrm{cm}^{2}$ up to $10 \mathrm{~T}$ at $4.2 \mathrm{~K}$ and $5 \mathrm{~T}$ at $20 \mathrm{~K}$. Simple wires, which achieve the properties of sintered bulk samples, are being made by the powder-in-tube route, indicating potential application capability, particularly if alloyed $\mathrm{MgB}_{2}$ wires replicating thin film properties can be made.
\end{abstract}

\section{CONSTRAINTS ON USE OF SUPERCONDUCTORS FOR MAGNETS}

The possibilities for application of any superconductor in a magnet are determined by the critical temperature $T_{c}$ and the upper critical field $H_{c 2}$. These thermodynamic quantities set the energy scale for the superconducting state, but $H_{c 2}$ can be significantly increased by reducing the electron mean free path. Increasing electron scattering by alloying, as by adding $47 \mathrm{wt} . \% \mathrm{Ti}$ to pure $\mathrm{Nb}$ produces little change in $\mathrm{T}_{\mathrm{c}}$ but enhances $H_{c 2}$ from $<1 \mathrm{~T}$ to about $12 \mathrm{~T}$ at $4.2 \mathrm{~K}$. Adding $1 \mathrm{wt} . \% \mathrm{Ti}$ to $\mathrm{Nb}_{3} \mathrm{Sn}$ raises $H_{c 2}$ by about $10 \%$. While both $\mathrm{Nb}$ and $\mathrm{Nb}_{3} \mathrm{Sn}$ are cubic and only weakly anisotropic, crystalline anisotropy can produce significant variation of $H_{c 2}$ in layered superconductors due to differences in electron transport with respect to the crystal symmetry axes. Untextured forms of anisotropic superconductors such as $\mathrm{MgB}_{2}$ and the high-temperature superconductors (HTS) are then limited by the lower value of $H_{c 2}$, that is for fields applied perpendicular to the superconducting planes $\left(H_{c 2 \perp}\right)$.

Practical limits for application of superconductors are further defined by the irreversibility field $H^{*}$, at which

\footnotetext{
*Work supported by the US Department of Energy, with additional
} facilities support from the US National Science Foundation. flux pinning is lost and the critical current density $J_{c}$ falls to zero. The maximum operating points for magnets are $\sim 0.8 H^{*}$ because of the need to have finite $J_{c}$ in the windings. The irreversibility fields of $\mathrm{Nb}-\mathrm{Ti}, \mathrm{Nb}_{3} \mathrm{Sn}$ and $\mathrm{MgB}_{2}$ as a function of temperature are shown in fig. 1 . Due to the finite thermal energy of the magnetic flux-line lattice, all three materials have $H^{*} 10-15 \%$ below $H_{c 2}$. $\mathrm{Nb} 47 \mathrm{Ti}$ magnets can thus reach $\sim 9 \mathrm{~T}$ at $4.2 \mathrm{~K}$ while $\mathrm{Nb}_{3} \mathrm{Sn}$ magnets can reach 20 to $22 \mathrm{~T}$ at $4.2 \mathrm{~K}$. In principle, HTS and Chevrel-phase conductors can reach still higher fields; for example, Bi-2212 conductors were recently used to make fields up to $23 \mathrm{~T}$ [1]. However, HTS conductors are limited by their anisotropy, and both HTS and Chevrel-phase conductors have processing difficulties. Conductor form matters-anisotropic conductors generally have better performance when made as tapes but such conductors are difficult to implement in accelerator magnets because there is no obvious way to make a fully transposed Rutherford cable from flat tapes. Thus round wire Bi-2212 is presently the only HTS material suitable for accelerator magnets.

Given a defining $H^{*}(T)$ envelope, the critical current density $J_{c}(H, T)$ becomes the index of superconductor performance because it determines the amount of conductor needed to achieve the required ampere-turns of the magnet. $J_{c}$ is determined by the vector force balance between flux pinning forces exerted by the microstructure on the flux lines and the Lorentz force of the applied current. Increasing the total pinning force, either by adding more pinning sites or by changing the mechanism by which magnetic flux interacts with material defects, results in higher $J_{c}$.

\section{CRITICAL FIELDS OF MAGNESIUM DIBORIDE}

The principal purpose of this paper is to discuss the potential for accelerator magnet application of the new superconductor $\mathrm{MgB}_{2}$. Since the compound was only reported to be superconducting in January 2001, much remains to be learned and any comments made at this time are subject to revision in light of a significant worldwide effort to understand its limits of performance. As indicated in fig $1, \mathrm{MgB}_{2}$ has a critical temperature of $39 \mathrm{~K}$ [2], more than double that of $\mathrm{Nb}_{3} \mathrm{Sn}$ and four times that of $\mathrm{Nb}-\mathrm{Ti}$, the present magnet materials. The crystal structure of $\mathrm{MgB}_{2}$ consists of close-packed $\mathrm{Mg}$ layers sandwiched between graphitic boron sheets . Due to this layered structure, there is a significant electronic anisotropy, which leads to anisotropic $H_{c 2}$ and $H^{*}$. The anisotropy ratio $H_{c 2||} / H_{c 2 \perp}$ ranges from 1.2 for aligned powders [17], 


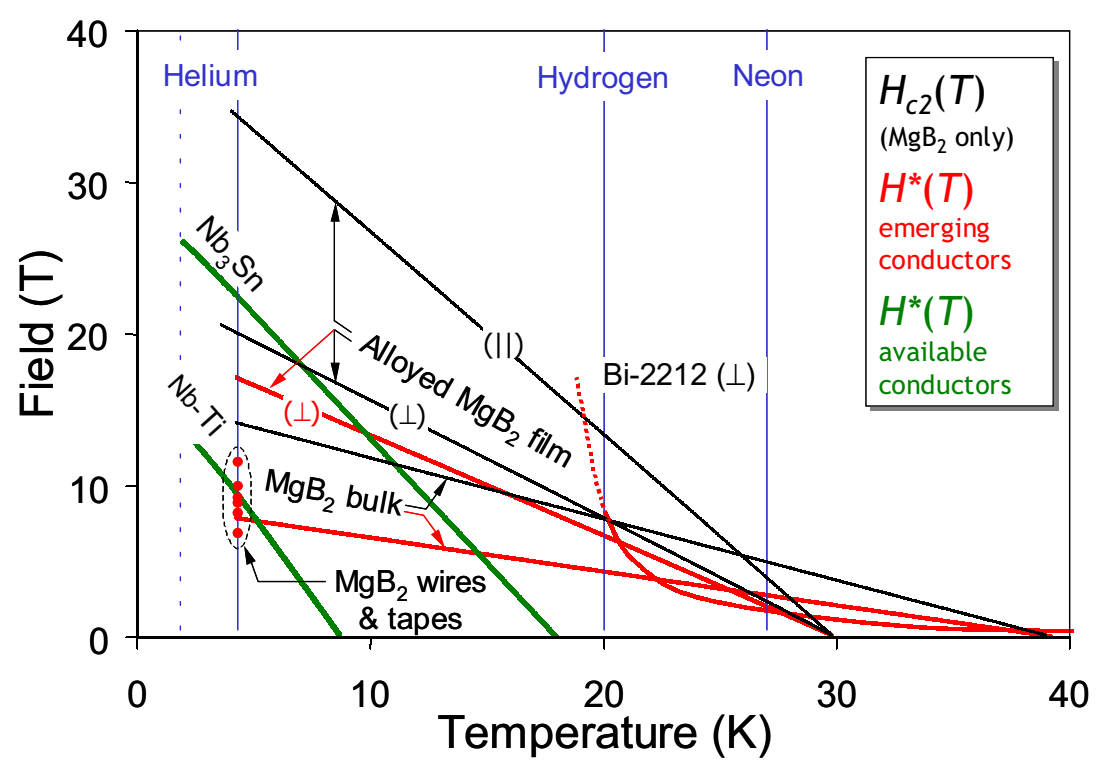

Fig. 1. Upper critical field $H_{c 2}$ (black lines) and irreversibility field $H^{*}$ (red and green lines) versus temperature for $\mathrm{MgB}_{2}$ compared to other magnet conductors. Optimized $\mathrm{Nb}$ - $\mathrm{Ti}$ and binary and alloyed $\mathrm{Nb}_{3} \mathrm{Sn}$ are indicated by the green lines. Data for $\mathrm{MgB}_{2}$ wires and tapes is presented at $4.2 \mathrm{~K}$ only (red dots). Also shown are isotherms for cryogens below $30 \mathrm{~K}$ (blue lines). The data for $\mathrm{Nb}$-Ti were taken from [3]; for $\mathrm{Nb}_{3} \mathrm{Sn}$ from [4]; for $\mathrm{Bi}-2212$ from [5]. The data for $\mathrm{MgB}_{2}$ came from [6] for films, [7]-[10] for bulk (see also fig. 2), and [11]-[16] for wires and tapes.

1.8 to 2.0 for textured thin films (fig. 1) [6], and up to 2.7 for single crystals [18], [19]. Here parallel refers to field aligned with the magnesium and boron layers.

In contrast to $\mathrm{Nb}-\mathrm{Ti}$ and $\mathrm{Nb}_{3} \mathrm{Sn}$, bulk samples and single crystals of $\mathrm{MgB}_{2}$ so far exhibit clean-limit behavior, with low values of resistivity, resistivity ratios significantly greater than 1 , and a small slope of $H_{c 2}(T)$ [7]-[10]. At $4.2 \mathrm{~K}$ the higher, that is parallel, upper critical field of bulk samples approaches $18 \mathrm{~T}$. In contrast, thin films have very small grains [20], [21], and can incorporate oxygen into the $\mathrm{MgB}_{2}$ layer [20]. Some films exhibit dirty-limit behavior, with high resistivity, resistivity ratios close to 1 , and a much steeper slope of $H_{c 2}(T)$ than is found in bulk samples. Upper critical field curves for bulk samples and c-axis textured, alloyed films are compared in fig. 1. In parallel field, $H_{c 2}(0)$ is as high as $39 \mathrm{~T}$ in the alloyed films, higher than the upper critical field of $\mathrm{Nb}_{3} \mathrm{Sn}$. However, the highest value in perpendicular field is about $22 \mathrm{~T}$.

Bulk samples are largely untextured and their irreversibility fields are about half of the parallel upper critical field, increasing at a rate of about $0.25 \mathrm{~T} / \mathrm{K}$ below $T_{c}$. At $4.2 \mathrm{~K}$, bulk samples have $H^{*}$ mostly $\sim 7 \mathrm{~T}$, fig. 1 . Wires and tapes also fall into this range. It is important to note that for these early samples the variation of $H^{*}$ indicated in fig. 1 is mostly due to the different electric field of the measurements, with higher $H^{*}$ values being determined by transport measurements than by magnetization measurements. Actual variations in superconducting properties are probably much less, and it will be important to develop a benchmark for comparing superconducting properties on a consistent basis. In addition, the large difference between $H_{c 2}$ and $H^{*}$ for bulk samples is a direct consequence of the lack of texture and the electronic anisotropy. $H^{*}(T)$ is defined by the first onset of dissipation, which occurs for those grains oriented perpendicular to the field, while $H_{c 2}(T)$ is defined by the loss of reversible superconducting magnetization in those grains whose planes are parallel to the applied field. In [22] it was shown that irradiation of bulk samples increases $H^{*}$, though it is unclear whether this is due to enhanced flux pinning or to enhanced scattering. The much higher upper critical field of alloyed thin films produces much higher values of $H^{*}$ in both parallel and perpendicular field. For applications, the lower perpendicular value $H^{*}$ defines the onset of dissipation and thus controls magnet design. Extrapolated transport data [6] indicate $H^{*}{ }_{\perp}$ is above $16 \mathrm{~T}$ at $4.2 \mathrm{~K}$ in these films.

An interesting result, shown in fig. 2, is that many bulk samples prepared by different routes in different laboratories exhibit identical $H_{c 2}(T)$ and $H^{*}(T)$. These common values of $H_{c 2}, T_{c}$ and $H^{*}$ suggest that there is little variation from $\mathrm{MgB}_{2}$ stoichiometry in the binary $\mathrm{Mg}-\mathrm{B}$ system. This is unlike $\mathrm{Nb}_{3} \mathrm{Sn}$ and other A-15 materials, which exhibit a range of composition and are susceptible to variations in superconducting properties brought about by a non-equilibrated diffusion reaction [4], [23].

\section{$3 J_{C}$ AND FLUX PINNING}

A fundamental issue addressed early on is the question of whether or not current flow between $\mathrm{MgB}_{2}$ grains is blocked by weak links, as it is for HTS materials. In [9], combined electron microscopy and magneto-optical imaging showed that induced supercurrents flowed 


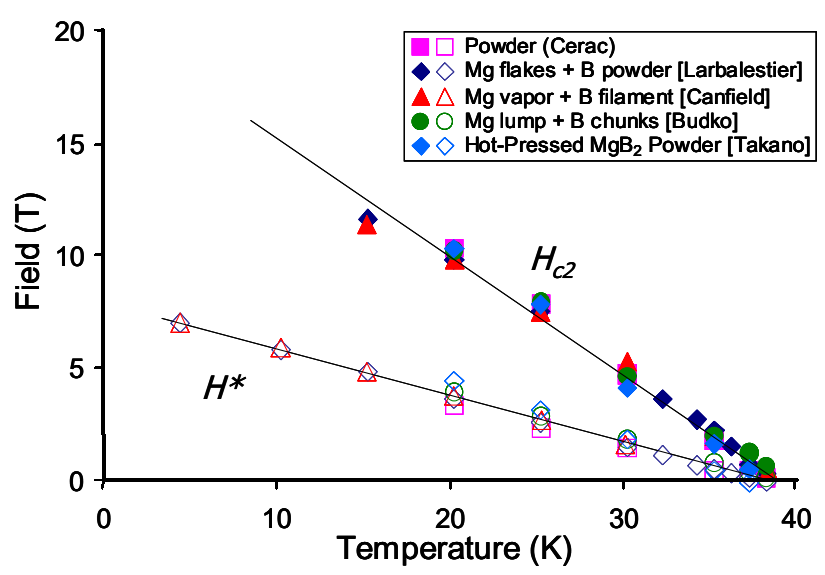

Fig. 2. Upper critical field and irreversibility field for several $\mathrm{MgB}_{2}$ samples prepared by different routes. All data were obtained from magnetization curves using a vibrating sample magnetometer. The values of $H_{c 2}(T)$ were determined by the disappearance of reversible magnetization, and the values of $H^{*}(T)$ were determined by extrapolations of Kramer plots to zero, as discussed in [9]. The lines are fits by eye.

around entire regions consisting of many tiny grains, indicating a lack of any weak links between the grains. Many subsequent studies have confirmed this conclusion [24]-[26]. Subsequent experiments [27] have found that it is even possible to obtain high $J_{c}$ values in powder-in-tube wires by rolling without subsequent sintering. Thus, on the whole $\mathrm{MgB}_{2}$ is a well-connected intermetallic superconductor more akin to $\mathrm{Nb}_{3} \mathrm{Sn}$ than to HTS. However, most present sample are less than fully dense and many contain insulating phases such as $\mathrm{MgO}$ and $\mathrm{B}_{2} \mathrm{O}_{3}$, which can form barriers between grains [21], [28]. $\mathrm{MgB}_{2}$ is sensitive to the environment and appropriate precautions for avoiding degradation are only now being worked out.

Magnetization measurements of early pressed bulk samples suggested that high $J_{c}$ was already present despite the inhomogeneity of samples. Using the dimensions of grain colonies, [9] deduced $J_{c}$ of $10^{5}$ to $10^{6} \mathrm{~A} / \mathrm{cm}^{2}$ at low fields and $4.2 \mathrm{~K}$ from VSM and magneto-optical measurements. Transport measurements carried out in [7] and [8] on samples with better homogeneity gave similar low-field $J_{c}$ values. A grain-boundary pinning mechanism was inferred [9] based on the temperature scaling and the shape of the bulk pinning force curve. Since this mechanism is also the dominant pinning mechanism in $\mathrm{Nb}_{3} \mathrm{Sn}$ composites, $J_{c}$ should increase inversely with grain size. Explicit proof of this hypothesis has not yet been provided, however.

Higher $J_{c}$ values have been reported in thin films [20], [29]-[32] where many results exceed $10^{6}$ to $10^{7} \mathrm{~A} / \mathrm{cm}^{2}$ in low field at $4.2 \mathrm{~K}$. Electron microscopy [21],[30] and other analyses [20] show that very small grains, as small as $10 \mathrm{~nm}$ in size, are produced in films made by pulsed laser deposition. The high number density of grain boundaries is consistent with such high $J_{c}$ values. In

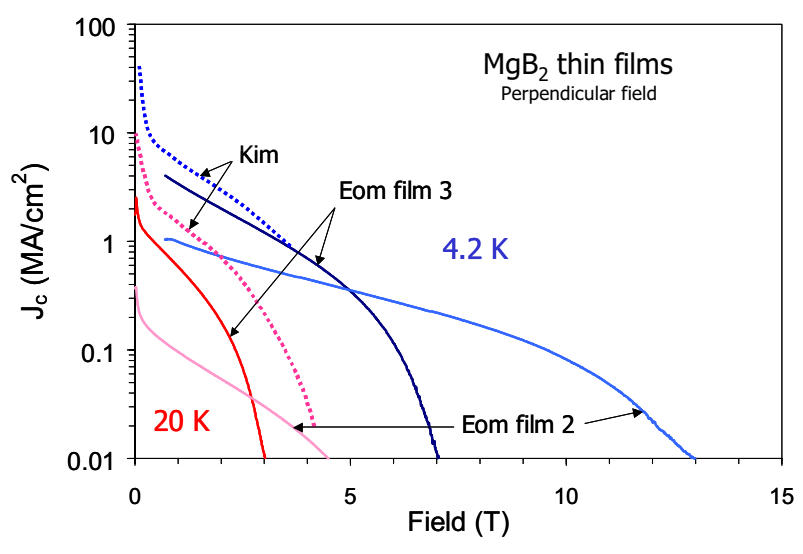

Fig. 3. Critical current density as a function of perpendicular applied field at 4.2 and $20 \mathrm{~K}$ for thin film $\mathrm{MgB}_{2}$ samples. These data were obtained from [20] and [29]. Magnetization measurements were used to determine the $J_{c}$ values.

addition, transmission electron microscopy shows a substantial fraction of insulating $\mathrm{MgO}$ particles $\sim 10 \mathrm{~nm}$ in size. These are likely additional flux-pinning centers, since the diameter of magnetic flux lines is about this size. In contrast to the magnetization data for bulk samples, the pinning-force curve did not show temperature scaling [20], perhaps because the fine-scale $\mathrm{MgO}$ pinning sites added to the flux pinning at lower temperatures when the particle size and the vortex core diameters $(\sim 7 \mathrm{~nm}$ at 4.2 $\mathrm{K})$ were comparable, raising $J_{c}$ values above $10^{5} \mathrm{~A} / \mathrm{cm}^{2}$ up to $10 \mathrm{~T}$ (perpendicular) field at $4.2 \mathrm{~K}$. Critical current density data demonstrating these points is shown in fig. 3 .

\section{$4 \mathrm{MgB}_{2}$ WIRES}

Within less than two weeks of the announcement of $\mathrm{MgB}_{2}$ as a superconductor, Canfield et al. [8] showed that $100-200 \mu \mathrm{m} \mathrm{MgB}_{2}$ filaments could be produced by reaction of $\mathrm{Mg}$ vapor with industrially available continuous boron filaments. Unfortunately, significant cracking occurs due to the large volumetric expansion of the filaments upon reaction has so far rendered this elegant route impractical.

More recent wire fabrication has used both pre-reacted $\mathrm{MgB}_{2}$ and mixed $\mathrm{Mg}$ and B powders in a powder-in-tube (PIT) process with a ductile metal sheath. A key issue is finding a suitable metal that will act as diffusion barrier and prevent the formation of harmful intermetallics with $\mathrm{Mg}$ or B and with a stabilizer such as $\mathrm{Cu}$. Jin et al. [33] pointed out that $\mathrm{Mo}, \mathrm{Nb}, \mathrm{Ta}, \mathrm{V}, \mathrm{W}, \mathrm{Hf}$ and $\mathrm{Fe}$ are suitable, Fe being cheap (although ferromagnetic too of course). Iron-boron compounds may be less stable than $\mathrm{MgB}_{2}$, which is also desirable. Jin et al. reported $4.2 \mathrm{~K}$, $1 \mathrm{~T} J_{c}$ values in excess of $10^{5} \mathrm{~A} / \mathrm{cm}^{2}$ and $20 \mathrm{~K}, 1 \mathrm{~T}$ values above $10^{4} \mathrm{~A} / \mathrm{cm}^{2}$ based on magnetization measurements, as shown in fig. 4.

Several other PIT designs have been investigated, and their $J_{c}$ properties are summarized in fig. 4. In [27], 


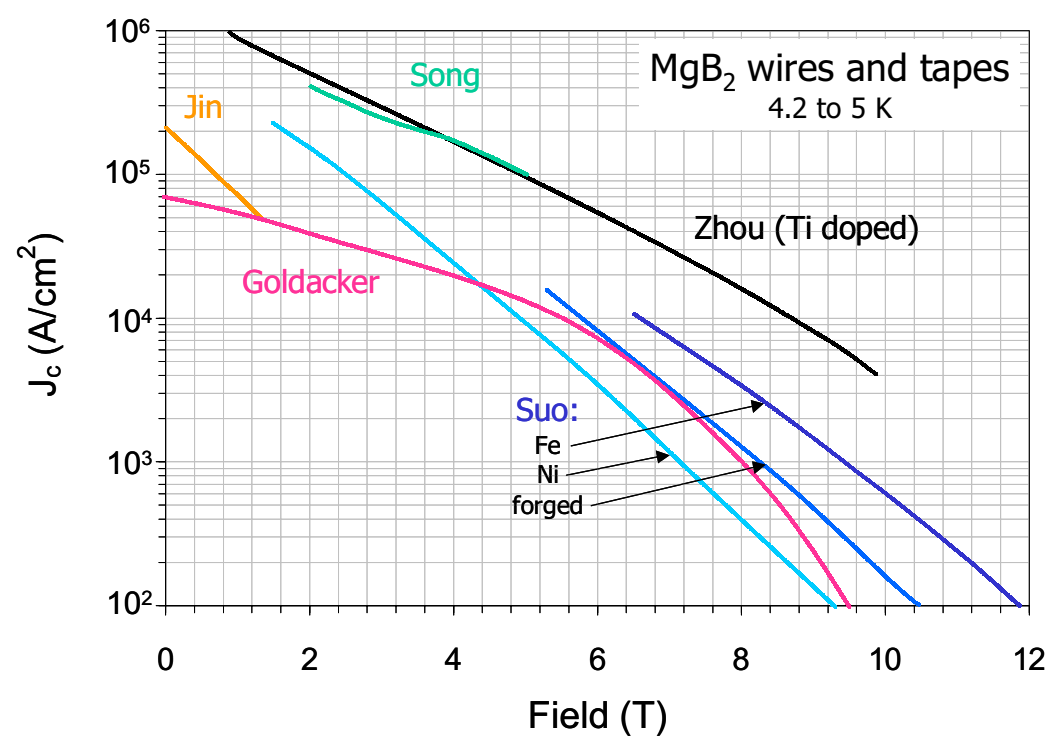

Fig. 4. Critical current density vs. applied field for $\mathrm{MgB}_{2}$ wires and tapes at a temperature of 4.2 or $5 \mathrm{~K}$. The upper 2 curves (Song, Zhao) were inferred from magnetization measurements, while the bottom curves (Suo, Goldacker, Jin) were obtained by direct transport measurements. These data were obtained from [11]-[15].

$\mathrm{MgB}_{2}$ powders were fabricated inside $\mathrm{Cu}, \mathrm{Ag}$, and $\mathrm{Ni}$ tubes without any diffusion barrier because good contact between grains could be obtained without sintering. Critical current densities above $10^{5} \mathrm{~A} / \mathrm{cm}^{2}$ were reported in self field at $4.2 \mathrm{~K}$. It also appears that the sheath may need to apply significant compressive stress to the powder core, as indicated by the correlation between sheath strength and $J_{c}$ in [27]. This point is also addressed in [11], where better performance was found for wires made with a Ta diffusion barrier and a reinforcing steel sheath outside the copper stabilizer. One reason for the better performance might be related to pulverization of the $\mathrm{MgB}_{2}$ powder during wire deformation. Since it is difficult to prevent surface contamination of $\mathrm{MgB}_{2}$ powder, fragmentation may be needed to expose the clean $\mathrm{MgB}_{2}$ surfaces needed for superconducting continuity.

It has also been reported that ball milling to reduce the $\mathrm{MgB}_{2}$ powder to submicron particle size is valuable in enhancing $J_{c}$ [12]. The best $J_{c}$ exceeds $10^{5} \mathrm{~A} / \mathrm{cm}^{2}$ at $4.2 \mathrm{~K}$ and $1.5 \mathrm{~T}$, and $10^{4} \mathrm{~A} / \mathrm{cm}^{2}$ at $4.2 \mathrm{~K}$ and $6.5 \mathrm{~T}$ was found for iron-sheathed tapes. A slight reduction in $T_{c}$ was also noted, to $37 \mathrm{~K}$. This may indicate a greater influence of electron scattering by grain boundaries in these wires and tapes, similar to what is observed in thin films. Very recently, doping with $\mathrm{Ti}$ was reported to improve the density of the $\mathrm{MgB}_{2}$ core, resulting in high magnetization current densities at $4.2 \mathrm{~K}$ [13]. These data are also included in fig. 4.

One of the potential difficulties already observed is the formation of a resistive barrier between the iron sheath and the $\mathrm{MgB}_{2}$ core. In [15], it was suggested that iron oxides on the inner surface of the sheath are reduced by either excess magnesium or by the decomposition of $\mathrm{MgB}_{2}$ into $\mathrm{Mg}$ vapor and higher magnesium-borides. This produced a large interface resistance and general difficulty in transferring current into the superconductor. As a whole there are still significant difficulties in making transport measurements on $\mathrm{MgB}_{2}$ wires and many more measurements have been made by magnetization than by transport. This sometimes leads to significant difference in $J_{c}$ magnitudes. This difference was explicitly examined in [16]. Exponents ( $n$-values) of the power-law resistive transition at the critical current, $E \propto\left(J / J_{c}\right)^{n}$, were usually less than 10. Since the electric field $E$ for inductive measurements, such as $\mathrm{dc}$ magnetization or ac susceptibility, is orders of magnitude below that of transport measurements, transport $J_{c}$ values can be a factor of 2 or more higher than inductive $J_{c}$ values. By contrast, high-quality $\mathrm{Nb}-\mathrm{Ti}$ and $\mathrm{Nb}_{3} \mathrm{Sn}$ strands have $n$ values of 50 or more, and $J_{c}$ values vary only by a few percent. One reason for the low $n$ values is the inhomogeneity of the current-carrying cross-section in these early strands. However, other work [22], [34], [35] raises concern that flux creep is rapid at high fields, which would exacerbate this effect in high-field magnets. As with the irreversibility field discussed earlier, a benchmark is needed to allow useful comparisons of critical current density data from different sources.

Conductor designs are still rather primitive, most being simple monofilament designs with core diameters of 100 $\mu \mathrm{m}$ or more. It is clear that the fabrication of the very hard $\mathrm{MgB}_{2}$ powder within softer metal tubes is not without problems, suggesting that the route to a multifilament conductor geometry will not be easy. It is worth remembering that accelerator conductors are amongst the most sophisticated ever developed, fine filaments of order $5-50 \mu \mathrm{m}$ being required in round wires that can withstand cabling into multikiloamp conductors. Flux-jump instabilities have been noted in several reports, 
reflecting the large filament diameters studied so far. Another potential problem, at least for magnetization evaluations, are ferromagnetic Fe and Ni sheaths, which must be removed prior to measurement. Due to the reactivity of $\mathrm{Mg}$, etching such barriers away is not yet possible.

\section{SUMMARY}

Although the research and development effort has barely begun, $\mathrm{MgB}_{2}$ already exhibits many favorable properties that suggest that it could become useful for magnet applications. Current flow between grains is not weak-linked, and so far no evidence for any intrinsic limitation to the current carrying capacity has emerged. The irreversibility field of bulk forms, wires, and tapes is comparable to that of Nb47wt.\%Ti alloy at liquid helium temperature, about $8 \mathrm{~T}$. Thin films suggest the potential for increasing this value to perhaps $16 \mathrm{~T}$ by alloying. Primitive powder-in-tube conductors attain at least $10^{5}$ $\mathrm{A} / \mathrm{cm}^{2}$ critical current densities in low fields at $4.2 \mathrm{~K}$, even without heat treatment to sinter the powder core together. Thin films suggest more than 10 times higher $J_{c}$ values might be obtained for wires or tapes with smaller grain size, full density, and perhaps added $\mathrm{MgO}$. In addition to these demonstrated results, the potential low cost and abundance of raw materials $(\mathrm{Mg}, \mathrm{B}, \mathrm{Cu}$, and $\mathrm{Fe}$ diffusion barriers) and the low density $\left(2.7 \mathrm{~g} / \mathrm{cm}^{3}\right)$ are attractive. Significant impact on magnet applications should result if $\mathrm{MgB}_{2}$ wires and tapes that at least partly replicate properties found in thin films can be produced.

Some of the work presented here would not be possible without the generous contribution of samples by $\mathrm{P}$ Canfield and S Budko (Ames), R Cava (Princeton), and H Kumakura (NIMS). Discussions with S Patnaik, J. Jiang, M Rzchowski, X Song, and X Cai are gratefully acknowledged.

\section{REFERENCES}

[1] T Kiyoshi et al., IEEE Trans. Appl. Supercond. 10:472 (2000)

[2] J Nagamatsu, N Nakagawa, T Muranaka, Y Zenitani, and J Akimitsu, Nature 410:63 (2001)

[3] PJ Lee Nb-Ti review; LD Cooley, PJ Lee, and DC Larbalestier, Chapter B3.3 of Handbook of Superconducting Materials, Institute of Physics, to appear.

[4] PJ Lee, AA Squitieri and DC Larbalestier, IEEE Trans. Appl. Supercond. 10: 979 (2000).

[5] Q Li, YN Tsay, M Suenaga, G Wirth, GD Gu and N Koshizuka, IEEE Trans. Appl. Supercond. 9:2026 (1999)
[6] S Patnaik et al., Supercond. Sci. Technol. 14:315 (2001)

[7] DK Finnemore, JE Ostenson, SL Bud'ko, G Lapertot, and PC Canfield, Phys. Rev. Lett. 86:2420 (2001)

[8] PC Canfield et al., Phys. Rev. Lett. 86:2423 (2001)

[9] DC Larbalestier et al, Nature 410:186 (2001)

[10] Y Takano et al, Appl. Phys. Lett. 78:2914 (2001)

[11]W Goldacker, SI Schlachter, S Zimmer, and $\mathrm{H}$ Reiner, preprint at http://xxx.lanl.gov/archive/condmat/0106226

[12]HL Suo et al., preprint at http://xxx.lanl.gov/archive/ cond-mat/0106341 and ICMC 2001

[13] Y Zhou et al., Appl. Phys. Lett. 79:1154 (2001)

[14] KJ Song et al., preprint at http://xxx.lanl.gov/archive/ cond-mat/0106124

[15] SX Dou et al., preprint at http://xxx.lanl.gov/archive/ cond-mat/0102320 and ICMC 2001

[16]M Dahlle et al., preprint at http://xxx.lanl.gov/ archive/ cond-mat/0104395

[17] OF de Lima, RA Ribeiro, MA Avila, CA Cardoso, and AA Coelho, Phys. Rev. Lett. 86:5974 (2001)

[18] M Xu et al., preprint at http://xxx.lanl.gov/archive/ cond-mat/0105271

[19] S Lee et al., preprint at http://xxx.lanl.gov/archive/ cond-mat/0105545

[20] CB Eom et al, Nature 411:558 (2001)

[21]X Song et al Submitted to Superconductor Science and Technology August 2001.

[22] Y Bugoslavsky et al., Nature 411:561 (2001)

[23] DC Larbalestier, LD Cooley, and PJ Lee, submitted to the $17^{\text {th }}$ Conference on Magnet Technology, 2001

[24] M Kambara et al., Supercond. Sci. Technol. 14:L5 (2001)

[25]Y Bugoslavsky, GK Perkins, X Qi, LF Cohen, and AD Caplin, Nature 410:563 (2001)

[26] A Polyanskii et al., Supercond. Sci. Technol. 14:811 (2001)

[27] G Grasso et al., Appl. Phys. Lett. 79:230 (2001)

[28] Y Zhu et al., preprint at http://xxx.lanl.gov/archive/ cond-mat/0105311

[29] HJ Kim et al., preprint at http://xxx.lanl.gov/archive/ cond-mat/0105363

[30] M Paranthaman et al., Appl. Phys. Lett. 78:3669 (2001)

[31] SR Shinde et al., Appl. Phys. Lett. 79:227 (2001)

[32] DH Blank et al., Appl. Phys. Lett. 79:394 (2001)

[33] S Jin, H Mavoori, and RB van Dover, Nature 411:563 (2001)

[34] MJ Qin, XL Wang, HK Liu, and SX Dou, preprint at http://xxx.lanl.gov/archive/cond-mat/0104112

[35] HH Wen et al., preprint at http://xxx.lanl.gov/archive/ cond-mat/0103521 